\title{
Histone deacetylase inhibitors induce remission in transgenic models of therapy-resistant acute promyelocytic leukemia
}

\author{
Li-Zhen He, ${ }^{1}$ Thomas Tolentino, ${ }^{2}$ Peter Grayson, ${ }^{2}$ Sue Zhong, ${ }^{1}$ Raymond P. Warrell, Jr., ${ }^{3}$ \\ Richard A. Rifkind, ${ }^{2}$ Paul A. Marks, ${ }^{2}$ Victoria M. Richon, ${ }^{2}$ and Pier Paolo Pandolfi ${ }^{1}$
}

\author{
${ }^{1}$ Molecular Biology Program and Department of Pathology, \\ ${ }^{2}$ Cell Biology Program, \\ ${ }^{3}$ Department of Medicine, Memorial Sloan-Kettering Cancer Center, Sloan-Kettering Division, \\ Graduate School of Medical Sciences, Cornell University, New York, New York, USA
}

Address correspondence to: Pier Paolo Pandolfi, Molecular Biology Program, Department of Pathology, Sloan-Kettering Institute, Memorial Sloan-Kettering Cancer Center, 1275 York Avenue, Box 110, New York, New York 10021, USA. Phone: (212) 639-6168; Fax: (212) 717-3102; E-mail: p-pandolfi@ski.mskcc.org.

\begin{abstract}
Acute promyelocytic leukemia (APL) is associated with chromosomal translocations, invariably involving the retinoic acid receptor $\alpha(R A R \alpha)$ gene fused to one of several distinct loci, including the $P M L$ or $P L Z F$ genes, involved in $\mathrm{t}(15 ; 17)$ or $\mathrm{t}(11 ; 17)$, respectively. Patients with $\mathrm{t}(15 ; 17)$ APL respond well to retinoic acid (RA) and other treatments, whereas those with $t(11 ; 17)$ APL do not. The PMLRAR $\alpha$ and PLZF-RAR $\alpha$ fusion oncoproteins function as aberrant transcriptional repressors, in part by recruiting nuclear receptor-transcriptional corepressors and histone deacetylases (HDACs). Transgenic mice harboring the $R A R \alpha$ fusion genes develop forms of leukemia that faithfully recapitulate both the clinical features and the response to RA observed in humans with the corresponding translocations. Here, we investigated the effects of HDAC inhibitors (HDACIs) in vitro and in these animal models. In cells from PLZF-RAR $\alpha / R A R \alpha-P L Z F$ transgenic mice and cells harboring $\mathrm{t}(15 ; 17)$, HDACIs induced apoptosis and dramatic growth inhibition, effects that could be potentiated by RA. HDACIs also increased RA-induced differentiation. HDACIs, but not RA, induced accumulation of acetylated histones. Using microarray analysis, we identified genes induced by RA, HDACIs, or both together. In combination with RA, all HDACIs tested overcame the transcriptional repression exerted by the RAR $\alpha$ fusion oncoproteins. In vivo, HDACIs induced accumulation of acetylated histones in target organs. Strikingly, this combination of agents induced leukemia remission and prolonged survival, without apparent toxic side effects.
\end{abstract}

J. Clin. Invest. 108:1321-1330 (2001). DOI:10.1172/JCI200111537.

\section{Introduction}

Acute promyelocytic leukemia (APL), the M3 subtype of acute myeloid leukemia (AML), accounts for more than $10 \%$ of all AMLs and is characterized by three distinctive and unique features (1-3): (a) the accumulation in the bone marrow (BM) of tumor cells with promyelocytic features; (b) the invariable association with specific reciprocal chromosomal translocations always involving the retinoic acid receptor $\alpha(R A R \alpha)$ gene on chromosome 17. The involvement of RAR $\alpha$, still to date one of the most well-studied transcription factors, in the pathogenesis of APL made this leukemia a straightforward example of aberrant transcription in cancer. As a consequence of the translocation, RAR $\alpha$ fuses to genes that have been recently identified (named PML, PLZF, NPM, NUMA, or STAT5b for brevity, and referred to as $X$ genes), leading to the generation of $R A R \alpha-X$ and $X$-RAR $\alpha$ fusion genes (3-12). The aberrant chimeric proteins encoded by these genes may exert a crucial role in leukemogenesis. APL blasts are sensitive to the differentiating action of retinoic acid (RA) $(1-3,13)$. RA can overcome the block of matura- tion at the promyelocytic stage and induce the malignant cells to terminally mature into granulocytes. However, treatment with RA in APL patients induces disease remission transiently, and relapse is inevitable $(1,2,13$, 14). Furthermore, APL associated with the translocation between the RAR $\alpha$ and the PLZF genes (PLZF$R A R \alpha$ ) has a poor prognosis compared with APL associated with PML-RAR $\alpha$, owing to a lack of response to either chemotherapy or treatment with RA (15).

RARs are members of the superfamily of nuclear hormone receptors that act as RA-inducible transcriptional activators in their heterodimeric form with retinoid$\mathrm{x}$-receptors (RXRs), a second class of nuclear retinoid receptors (16). In the absence of RA, RAR/RXR can repress transcription through histone deacetylation by recruiting nuclear receptor corepressors $(\mathrm{N}-\mathrm{CoR}$ or SMRT), Sin3A or Sin3B, in turn associating with histone deacetylases (17-19), thereby resulting in nucleosome assembly and transcriptional repression (19). RA causes the dissociation of the corepressor complex and the recruitment of transcriptional coactivators to the $\mathrm{RAR} / \mathrm{RXR}$ complex. This is associated with terminal dif- 
ferentiation and growth arrest of various types of cells, including normal myeloid hemopoietic cells (18-21).

We and others have proposed a model for the mechanisms of transcriptional repression by the X-RAR $\alpha$ fusion protein, which can explain both the molecular pathogenesis of APL and the differential response to RA (22-24). Our analysis of transgenic mice (TM) in which the PML-RAR $\alpha$ and PLZF-RAR $\alpha$ fusion genes are specifically expressed in the promyelocytic cellular compartment has revealed that the X-RAR $\alpha$ fusion proteins play a critical role in leukemogenesis and in determining responses to RA. PLZF-RAR $\alpha$ mice develop RA-resistant leukemia, whereas PML-RAR $\alpha$ mice develop APL-like leukemias that respond to RA $(22,25)$. We have demonstrated that both PML-RAR $\alpha$ and PLZF-RAR $\alpha$ can act as transcriptional repressors and are able to interact with nuclear receptor transcriptional corepressors such as SMRT and N-CoR (22-24). PML-RAR $\alpha$ can act as a dominant negative transcriptional repressor of RAR $\alpha$, through a nuclear corepressor association that is less sensitive to RA than is the RAR $\alpha /$ nuclear corepressor association. PLZF-RAR $\alpha$ can form, also via its PLZF moiety, corepressor complexes that are insensitive to RA (22). Histone deacetylase inhibitors (HDACIs) such as trichostatin A (TSA), in combination with RA, can overcome the transcriptional repressive activity of PMLRAR $\alpha$ and PLZF-RAR $\alpha$ (22-24). These observations suggest that HDACIs alone or in combination with RA may be useful in the therapy of APL.

Several classes of HDACIs have been identified, including: (a) short-chain fatty acids (26) (e.g., butyrates); (b) organic hydroxamic acids $(27,28)$ (e.g., TSA and hybrid polar compounds [HPCs]); (c) cyclic tetrapeptides containing a 2-amino-8-oxo 9,10-epoxydecanoyl (AOE) moiety (27) (e.g., trapoxin); and (d) cyclic peptides not containing the AOE moiety $(27,29$, 30) (e.g., FR901228, apicidin). Phenylbutyrate has been used as a single agent in the treatment of $\beta$-thalassemia, toxoplasmosis, and malaria. It has been successfully used by our group in the treatment of one patient with refractory APL in combination with RA (31), although four other patients with refractory APL were unresponsive to the same therapeutic regimen (32). The butyrates are not ideal agents for clinical use owing to the high concentrations required (mM) to achieve inhibition of HDAC activity, lack of specificity for inhibition of HDAC, and the low rate of effective response. Hydroxamic acid-based HPCs are low molecular weight synthetic compounds that have in common two polar groups separated by a hydrophobic five- or six-carbon methylene chain. These HPCs are active at micromolar concentration as inducers of cell differentiation and/or apoptosis $(28,33)$.

This study was designed to define the biologic and therapeutic effects of HDACIs in APL. In particular, we have analyzed the mechanisms of action of HDACIs and RA, the consequences of these treatments on gene expression in APL blasts, and their effects on the aberrant transcriptional activity of the X-RAR $\alpha$ fusion pro- teins. Furthermore, we have tested the efficacy of HDACIs in vivo, in TM that coexpress PLZF-RAR $\alpha$ and RAR $\alpha$-PLZF proteins in the myeloid-promyelocytic cellular compartment, thus recreating the dual complexity of APL genetics. PLZF-RAR $\alpha / R A R \alpha-P L Z F$ double TM develop leukemia at 100\% penetrance by 6 months of age. These leukemias display features of APL, such as a dramatic accumulation of immature blasts and of cells blocked at the promyelocytic stage of differentiation, which is distinctive of human APL (34). Furthermore, leukemia in these mice is markedly resistant to $\mathrm{RA}$ and $\mathrm{As}_{2} \mathrm{O}_{3}$ treatment $(34,35)$. Based on these observations, preclinical trials were performed in this unique therapy-resistant mouse model of APL using SAHA in combination with RA. This in vivo analysis showed that SAHA and RA in combination are effective in prolonging survival and inducing disease remission.

\section{Methods}

Cell cultures. NB4 (an APL cell line that expresses the PML-RAR $\alpha$ fusion protein) was maintained in RPMI 1640 medium supplemented with $10 \% \mathrm{FBS}, 2 \mathrm{mM}$ L-glutamine, and $1 \%$ penicillin-streptomycin, at $37^{\circ} \mathrm{C}$ in $5 \%$ $\mathrm{CO}_{2}$ atmosphere. Cultures were set up at an initial density of $10^{5}$ cells $/ \mathrm{ml}$ in the presence or absence of various concentrations of PB (kindly supplied by Adria-sp Inc. for Elan Pharmaceutical Research Corp., Gainesville, Georgia, USA and the Cancer Treatment Evaluation Program, National Cancer Institute, Bethesda, Maryland, USA); TSA (Wako Pure Biochemicals, Richmond, Virginia, USA), or SAHA, synthesized as described previously (28), with or without $10^{-6} \mathrm{M}$ of all-trans RA (Sigma Chemical Co., St. Louis, Missouri, USA).

$\mathrm{BM}$ cells isolated from PLZF-RAR $\alpha / R A R \alpha-P L Z F$ double TM with leukemia, as well as wild-type mice, were cultured in parallel in DMEM supplemented with 20\% FBS, $2 \mathrm{mM}$ L-glutamine, and $1 \%$ penicillin-streptomycin at $37^{\circ} \mathrm{C}$ in $5 \% \mathrm{CO}_{2}$ atmosphere. Cultures were set up at a cell density of $2 \times 10^{6} / \mathrm{ml}$ with or without $2 \%$ pokeweed mitogen-stimulated spleen cell-conditioned media (SCCM) containing IL3 and GM-CSF (StemCell Technologies, Vancouver, British Columbia, Canada). SAHA $(0.6 \mu \mathrm{M})$ and RA $\left(10^{-6} \mathrm{M}\right)$ were added as indicated for each experiment.

Proliferation assays. For $\left[{ }^{3} \mathrm{H}\right]$ thymidine incorporation assay, $180-\mu \mathrm{l}$ aliquots from each culture were placed into 96-well plates in triplicate after 20 hours of incubation with or without PB, TSA, or SAHA and/or RA, and $0.5 \mu \mathrm{Ci}$ of $\left[{ }^{3} \mathrm{H}\right]$ thymidine in $20 \mu \mathrm{l}$ of medium was added into each well. After further incubation for 24 hours, cells were harvested onto glass fiber filter and placed in a liquid scintillation counter (1450 Microbeta Plus; filter and counter both from Wallac, Turku, Finland). For growth curves, viable cells were scored each day up to 4 days by trypan blue exclusion from triplicate cultures.

Cell-cycle analysis. After 20 hours of incubation with various drug combinations, cells were harvested, fixed with $1 \%$ paraformaldehyde, treated with $0.1 \%$ RNAse A, 
stained with propidium iodide (PI), and subsequently analyzed by flow cytometry (Becton Dickinson \& Co., Franklin Lakes, New Jersey, USA) using Cell Quest and ModFit LF softwares (Becton Dickinson \& Co.).

Apoptosis. The proportion of cells undergoing apoptosis was determined by TUNEL and by Annexin V staining (36). Aliquots $(1 \mathrm{ml})$ from each culture were harvested after 24 and 48 hours of incubation for TUNEL and Annexin V analysis, respectively. Cytospun cells were stained with a TUNEL kit following the manufacturer's directions (Roche Molecular Biochemicals, Indianapolis, Indiana, USA). 4',6-diamidino-2-phenylindole (DAPI) was used to reveal nuclei. For each point, at least 300 cells were scored under a fluorescence microscope (Olympus, Middlebush, New Jersey, USA). Cells were also stained with FITC-conjugated Annexin V and PI, according to the manufacturer's instruction (PharMingen, San Diego, California, USA), and analyzed by flow cytometry.

Differentiation assays. Cellular differentiation was studied as described previously $(22,25)$ upon 4 days of culture with the various drugs using both a nitroblue tetrazolium (NBT) reduction assay and also by analyzing the expression of $\mathrm{CD} 11 \mathrm{~b}$, a cell-surface differentiation antigen.

Immunofluorescence staining. NB4 cells were cytospun on slides after 24 hours of incubation with the different drugs and drug combinations. For staining of acetylated histone, slides were fixed in $95 \%$ ethanol $/ 5 \%$ acetic acid for 1 minute, permeabilized, and blocked in $10 \%$ goat serum $/ 1 \%$ BSA $/ 0.3 \%$ Triton X-100/PBS for 40 minutes. The slides were then incubated with antibodies that specifically recognize the acetylated forms of histone $\mathrm{H} 3$ and histone $\mathrm{H} 4$ (Upstate Biotechnology Inc., Lake Placid, New York, USA) for 1 hour. Staining for the PML protein was carried out as described previously $(22,25)$. A Texas red-conjugated IgG was used as secondary antibody. Staining with DAPI was carried out to reveal nuclei.

Western analysis of isolated acetylated bistones. Nuclei from NB4 cells were isolated by lysis in buffer containing $10 \mathrm{mM}$ Tris- $\mathrm{HCl}$ ( $\mathrm{pH} 6.5$ ), $50 \mathrm{mM}$ sodium bisulfite, $1 \%$ Triton $\mathrm{X}-100,10 \mathrm{mM} \mathrm{MgCl} 2,8.6 \%$ sucrose, and Dounce homogenization. Histones were isolated by acid extraction as described previously (28). Isolated histones $(5 \mu \mathrm{g})$ were then separated on 15\% SDS-PAGE minigels (Bio-Rad Laboratories Inc., Hercules, California, USA) and transferred to nitrocellulose (Schleicher and Schuell, Keene, New Hampshire, USA). Acetylated histones were detected utilizing the aforementioned anti-acetylated histone $\mathrm{H} 3$ and histone $\mathrm{H} 4$ antibodies and were visualized by chemiluminescence (Pierce Chemical Co., Rockford, Illinois, USA). As a control for the amount of protein loading, gels run in parallel were stained with Coomassie blue.

cDNA microarray analysis. Total RNA from NB4 cells cultured for 6 hours with RA $(1 \mu \mathrm{M})$ and SAHA $(0.9$ $\mu \mathrm{M})$ alone and in combination was isolated using the guanidine isothiocyante/phenolchloroform method (37). Poly A+ RNA was isolated using the Oligotex
mRNA kit from QIAGEN Inc. (Valencia, California, USA). Poly A+ RNA (600 ng) was sent to Incyte Genomics Inc. (Palo Alto, California, USA) for expression analysis using the Incyte Human UniGEM V 2.0 cDNA array (Incyte Genomics Inc., Palo Alto, California, USA). Probe generation and expression analyses were performed by Incyte Genomics Inc. Analysis for specific gene expression was carried out using the GemTools Analysis software (Incyte Genomics Inc.). Changes in gene expression less than twofold were not considered significant.

Northern analysis. Total RNA from NB4 cells was isolated using the guanidine isothiocyanate/phenol/chloroform method (37). RNA $(10 \mu \mathrm{g})$ was electrophoresed through a $1 \%$ agarose gel containing $17 \%$ formaldehyde. The RNA was blotted onto Hybond-N membranes (Amersham Pharmacia Biotech Inc., Piscataway, New Jersey, USA) using standard techniques, and the blots were hybridized with cDNA probes. The following probes were used: 2.12-kB p21WAF1 cDNA fragment from $\mathrm{pZL}$ WAF1 (38); 18S rRNA-specific 50-mer oligodeoxyribonucleotide that was end labeled with $\left[{ }^{\gamma 32} \mathrm{P}\right]-\mathrm{ATP}$ by using T4 polynucleotide kinase (39); and G0S2, transglutaminase 2 , integrin $\beta 7$, and thymidine kinase I(TK1) that were obtained from Genome Systems Inc. (St. Louis, Missouri, USA) and sequence verified.

Transactivation assays. Cos- 1 cells were transfected with a DR5-Luciferase reporter; Tk- $\beta$-galactosidase internal standard; and PSG5, pSG5-RAR $\alpha$, pSG5-PML-RAR $\alpha$, or PSG5-PLZF-RAR $\alpha$ expression vectors using the SuperFect Transfection Regent (QIAGEN Inc., Santa Clarita, California, USA). Transfected cells were then incubated with $4 \mathrm{mM} \mathrm{PB}, 30 \mathrm{nM}$ TSA, or $0.9 \mu \mathrm{M}$ SAHA alone or in combination with $10^{-7} \mathrm{M}$ RA for 48 hours. The aforementioned concentrations of HDACIs were chosen for the transcriptional assays because higher doses resulted in toxic for Cos- 1 cells. Luciferase activity of each sample was measured in a luminometer (Turner Designs, Sunnyvale, California, USA) and normalized to $\beta$-galactosidase activity.

In vivo studies with SAHA in mice. For toxicity studies, wild-type mice were daily injected intraperitoneally with SAHA (20-200 $\mu \mathrm{g} / \mathrm{g}$ mouse body weight) alone and in combination with RA $(6 \mu \mathrm{g} / \mathrm{gbw})$ for 28 days. Automatic and differential counting of peripheral blood was performed weekly. The mice were kept for a subsequent 6month follow-up, or sacrificed for pathological analysis. For Western blot analysis of histones acetylation, peripheral blood, BM, and spleen cells from mice were collected before and 2 hours after one injection of SAHA at a dose of $20 \mu \mathrm{g} / \mathrm{gbw}$. Isolation of histones and Western blot analysis was performed as aforementioned.

In vivo therapeutic trials were carried out in PLZF$R A R \alpha / R A R \alpha-P L Z F$ leukemic TM. Mice were bled via tail biweekly to monitor the peripheral blood for the presentation of leukemia according the following criteria: in two consecutive peripheral blood samples, if (a) Myeloblast or promyelocyte appeared; (b) White blood cell $(\mathrm{WBC})>30 \times 10^{3} / \mu \mathrm{l}$, plus $\mathrm{Hb} \downarrow(<10 \mathrm{~g} / \mathrm{dl})$, 
a
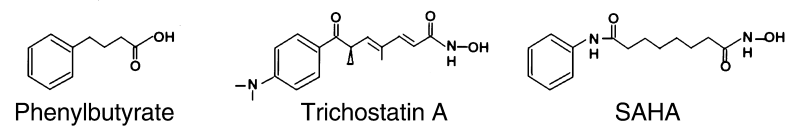

b
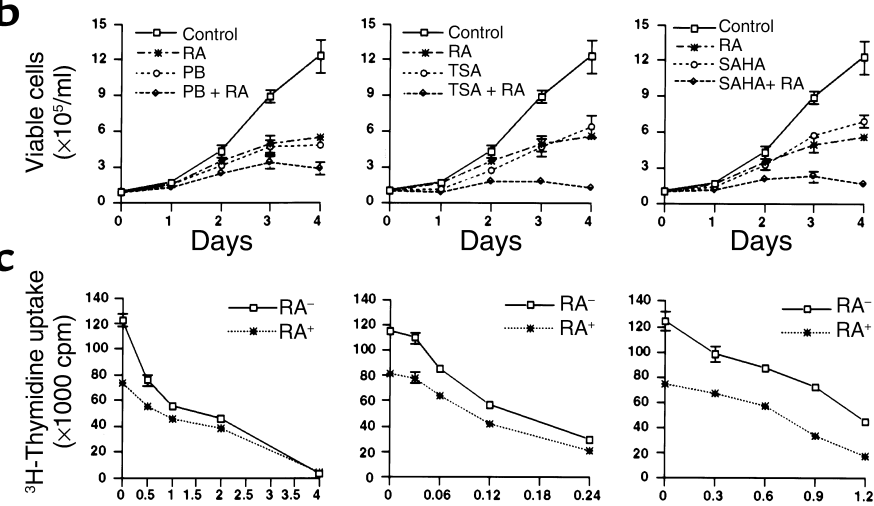

PB (mM)

d

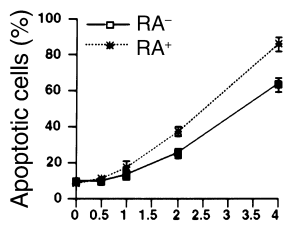

PB (mM)

e

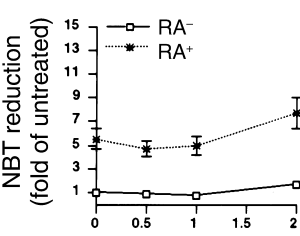

PB (mM)

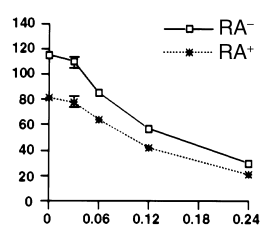

TSA $(\mu \mathrm{M})$

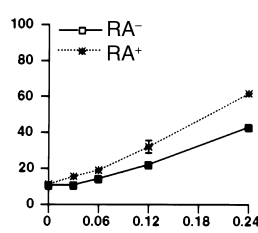

TSA $(\mu \mathrm{M})$

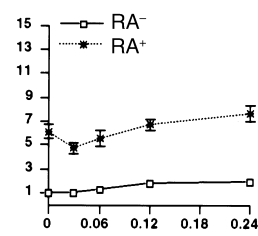

TSA $(\mu \mathrm{M})$

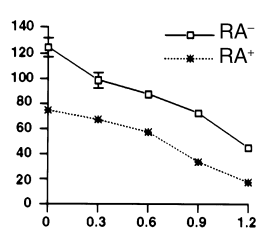

SAHA $(\mu M)$

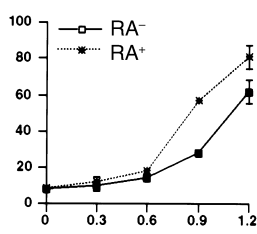

SAHA $(\mu M)$

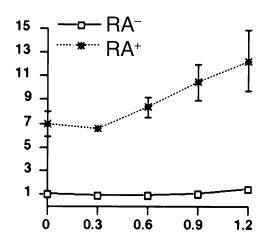

SAHA $(\mu \mathrm{M})$

\section{Figure 1}

The effects of HDACls alone and in combination with RA on NB4 cell growth, apoptosis, and differentiation. (a) Structures of the HDACls utilized in this study. (b) NB4 cells cultured at an initial cell density of $10^{5} / \mathrm{ml}$ in the presence of PB, TSA, or $\mathrm{SAHA}$ alone and in combination with RA $\left(10^{-6} \mathrm{M}\right)$. Growth curves were performed on NB4 cells cultured with $1 \mathrm{mM} \mathrm{PB}$, $0.12 \mu \mathrm{M}$ TSA, or $0.6 \mu \mathrm{M}$ SAHA alone or in combination with RA. (c) ${ }^{3} \mathrm{H}$-Thymidine incorporation was assayed after 20 hours' culture with PB, TSA, or SAHA at the indicated concentrations and in combination with $\mathrm{RA}\left(10^{-6} \mathrm{M}\right)$ and a further 24 hours with ${ }^{3} \mathrm{H}$-Thymidine. Each point represents results from three independent experiments performed in triplicate for $\mathbf{b}$ and $\mathbf{c}$ (mean \pm SD). (d) The percentage of apoptotic cells was measured after 48 hours' culture by using flow cytometry analysis of Annexin V-labeled cells. Each point represents results from three independent experiments performed in duplicate (mean $\pm \mathrm{SD}$ ). (e) The NBT reduction assay was performed after 4 days of culture and measured as $\mathrm{OD}_{540 \mathrm{~nm}} / 10^{6}$ cells. Each point represents results from three independent experiments performed in duplicate (mean $\pm S D$ ). The $\mathrm{OD}_{540 \mathrm{~nm}}$ value induced by various $\mathrm{HDACl}$ s with or without $10^{-6} \mathrm{M} \mathrm{RA}$ normalized to untreated control (untreated $=1$ ).
Plt $\downarrow\left(<800 \times 10^{3} / \mu \mathrm{l}\right) ;(\mathrm{c}) \mathrm{Hb}<6 \mathrm{~g} / \mathrm{dl}$ and Plt $<500 \times$ $10^{3} / \mu \mathrm{l}$; or $(\mathrm{d}) \mathrm{Hb}<3 \mathrm{~g} / \mathrm{dl}$ or Plt $<300 \times 10^{3} / \mu \mathrm{l}$. Once leukemia was diagnosed, therapy with RA was commenced. Mice were treated with RA for 2 weeks and then either continued on RA alone or changed to SAHA alone or RA in combination with SAHA for 4 weeks. RA was administered per orally daily at a dose of $1.5 \mu \mathrm{g} / \mathrm{gbw}$ and SAHA by daily intraperitoneal injection at $50 \mu \mathrm{g} / \mathrm{gbw}$. During the treatment, mice were bled once a week, and WBC, Hb, platelet, and differential counts were monitored in order to evaluate the response to the different therapies. Untreated control groups consisted of leukemic mice from the same transgenic lines that were also bled once a week. A Kaplan Meier curve was used for the survival analysis.

\section{Results}

HDACIs cause growth inbibition, apoptosis, and potentiate $R A$-induced differentiation in APL cells harboring $t(15 ; 17)$. We tested the effects of HDACIs (Figure 1a) on the NB4 cell line that was derived from an APL patient that harbors the $t(15 ; 17)$ and responds to the differentiating activity of RA. Growth curves and $\left[{ }^{3} \mathrm{H}\right]$ thymidine incorporation demonstrated a potent dose-dependent growth inhibitory activity of the three HDACIs (Figure $1, \mathrm{~b}$ and $\mathrm{c}$ ). As previously shown, RA markedly inhibit- ed NB4 cell growth. Combinations of HDACIs and RA exerted additive growth inhibitory effects.

Cell-cycle analysis of NB4 cells revealed that HDACIs as well as RA caused a decrease in the proportion of cells in S-phase (Table 1). This was accompanied by an increase of the G0/G1 fraction in the cells treated with either RA or HDACIs, whereas HDACIs also induced an increase of the $\mathrm{G} 2 / \mathrm{M}$ population. We did not observe additive effects on cell-cycle distribution when the cells were treated with combinations of HDACIs and RA, except that SAHA-RA combinations caused the greatest increase in the G2/M fraction (Table 1). Thus, HDACIs

\section{Table 1}

Effects of HDACls on cell cycle progression in NB4 cells

\begin{tabular}{lccc}
\cline { 2 - 2 } Treatment & G0/G1\% & S \% & G2/M \% \\
Control & $30.97 \pm 0.91$ & $65.81 \pm 5.03$ & $3.22 \pm 0.80$ \\
RA & $50.16 \pm 0.64$ & $47.91 \pm 1.74$ & $1.93 \pm 0.62$ \\
PB & $56.83 \pm 1.73$ & $36.20 \pm 2.35$ & $6.98 \pm 0.77$ \\
PB+RA & $64.11 \pm 1.28$ & $29.22 \pm 0.26$ & $6.66 \pm 1.03$ \\
TSA & $60.11 \pm 4.57$ & $24.00 \pm 5.02$ & $15.89 \pm 2.89$ \\
TSA+RA & $46.24 \pm 1.19$ & $38.95 \pm 1.76$ & $14.80 \pm 2.80$ \\
SAHA & $58.42 \pm 4.22$ & $27.12 \pm 3.23$ & $14.47 \pm 1.15$ \\
SAHA+RA & $48.36 \pm 1.41$ & $28.67 \pm 0.70$ & $23.40 \pm 1.67$
\end{tabular}

NB4 cells were cultured as described in Figure 1b, treated with RNAse, stained with $\mathrm{PI}$, and analyzed by flow cytometry. The results are expressed as mean \pm SD from one representative experiment performed in triplicate. 


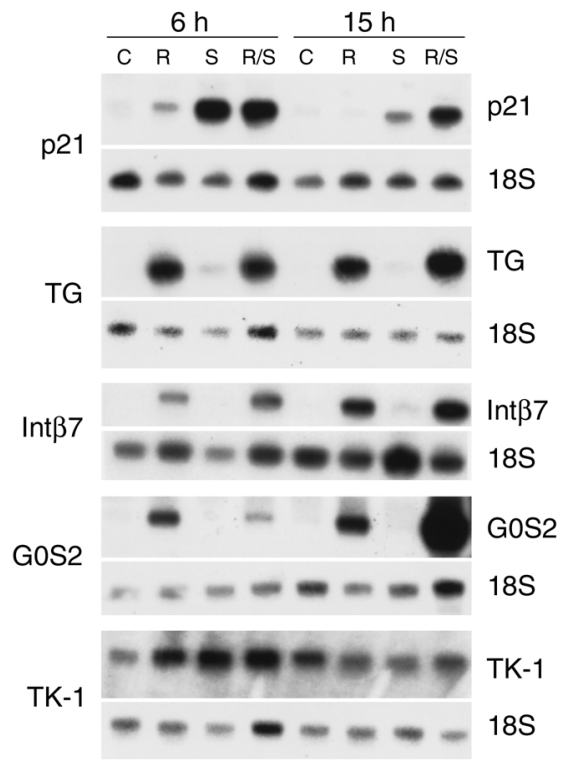

Figure 2

Northern blot analysis of potential RA and SAHA target genes after culture of NB4 cells with RA $(1 \mu \mathrm{M})$, SAHA $(0.9 \mu \mathrm{M})$, and the combination of RA and SAHA for the indicated times. Total RNA $(10 \mu \mathrm{g})$ was isolated and fractionated on $1.0 \%$ agarose/formaldehyde and transferred to a nylon membrane. The membrane was probed by using ${ }^{32}$ P-random labeled cDNA probes as indicated. As a loading control, the same membranes were also probed with a 50-mer oligonucleotide specific for $18 \mathrm{~S}$ rRNA.

or HDACIs-RA combinations display potent growth inhibitory effects in NB4 cells. This growth-suppressive activity can be attributed, at least in part, to a delay or arrest in the transition through the cell cycle.

To determine whether HDACIs-mediated cell growth inhibition is associated with the induction of apoptosis, we performed Annexin V labeling (Figure 1d) and TUNEL assay (data not shown) in NB4 cells treated with HDACIs and HDACIs-RA combinations. This analysis showed that the three HDACIs induced apoptosis in a dose-dependent manner. Although RA alone did not increase the spontaneous rate of apoptosis, it potentiated the proapoptotic activity of the three HDACIs in NB4 cells.

We next studied the differentiating activity of HDACIs in NB4 cells. Terminal differentiation of NB4 cells by the three compounds was negligible, as assayed by nitroblue tetrazolium (NBT) reduction (Figure 1e) and expression of the CD11b cell surface marker (data not shown). However, HDACIs potentiated RA-induced differentiation to different extents even at pharmacological dose of RA $\left(10^{-6} \mathrm{M}\right)$ (Figure 1e). The three HDACIs, but not RA, induced detectable accumulation of acetylated histones $\mathrm{H} 3$ and $\mathrm{H} 4$ in NB4 cells (data not shown). Thus, HDACIs display a growth- inhibitory and proapoptotic activity and have the ability to potentiate differentiation induced by pharmacological dose of RA even in cells that would normally respond to RA.

Induction of gene expression by RA and SAHA in NB4 cells. To identify genes whose expression is induced by RA and SAHA, we have carried out cDNA microarray analysis using RNA isolated after culture of NB4 cells with RA and SAHA alone and in combination for 6 hours (see Methods). The UniGem V 2.0 array contains 8,502 unique genes and expressed sequence tags (ESTs). At first, we analyzed the array for genes that have previously been identified as RAR $\alpha$ target genes and which have been demonstrated to mediate RAinduced differentiation (i.e., transglutaminase 2 [TG]) (40) and growth inhibition (i.e., $\mathrm{p} 21^{\mathrm{WAF} 1}$ ). TG was induced 5.9-fold by array, was not induced by SAHA alone, and was found to be induced 10.3 -fold by the combination of RA and SAHA. p21 WAF1 was not induced by RA alone, was induced 4.3 -fold by SAHA, and 4.4 -fold by the combination. To validate the array at 6 hours and examine the effect on gene expression at later time points (15 hours), Northern blot analysis was performed on NB4 as indicated (Figure 2). This analysis confirmed the array data at 6 hours. Furthermore, at 15 hours, we found that the combination of RA and SAHA markedly potentiated $\mathrm{p} 21^{\mathrm{WAF} 1}$ expression. Further analysis revealed that 60 genes were induced greater than twofold in NB4 cells cultured with RA; 48 genes were induced greater than twofold after culture with SAHA; and 80 genes were induced greater than twofold with the combination of RA and SAHA. The combination of RA and SAHA results predominantly in the induction of a combination of both the RA-induced genes and the SAHA-induced genes. Of the genes that were induced, there is very little overlap between the two treatments (RA and SAHA) with only two genes (thymidine kinase 1 and proteosome subunit $\beta$ type, 9) being independently induced by RA alone, SAHA alone, and the combination. Approximately $3 \%$ of the genes are induced by both RA alone and SAHA alone. In addition, nine genes were identified as being induced two- to threefold by the combi-
Table 2

Upregulation of genes by the combination of RA and SAHA in NB4 cells

$\begin{array}{llccc}\begin{array}{l}\text { GenBank } \\ \text { Accession Number }\end{array} & \text { Description } & \begin{array}{c}\text { RA } \\ \text { (fold) }\end{array} & \begin{array}{c}\text { SAHA } \\ \text { (fold) }\end{array} & \begin{array}{c}\text { RA+SAHA } \\ \text { (fold) }\end{array} \\ \text { X75314 } & \text { seb4D } & n s^{*} & n s & 2.7 \\ \text { AC004770 } & \text { hypothetical protein } & n s & n s & 2.5 \\ \text { AF258341 } & \text { P450 cytochrome oxidoreductase } & n s & n s & 2.4 \\ \text { AW955122 } & \text { (syn)hypothetical protein } & n s & n s & 2.3 \\ \text { AF016268 } & \text { death receptor 5 } & n s & n s & 2.3 \\ \text { NM_000041 } & \text { apolipoprotein E } & n s & n s & 2.2 \\ \text { X55448 } & \text { glucose 6-phosphate dehydrogenase } & n s & n s & 2.2 \\ \text { M34065 } & \text { cell division cycle 25C } & n s & n s & 2.0 \\ \text { Al554560 } & \text { GTP binding protein Rho7 } & n s & n s & 2.0\end{array}$

${ }^{*} n s$ : indicates that the change expression level is below 2-fold. 


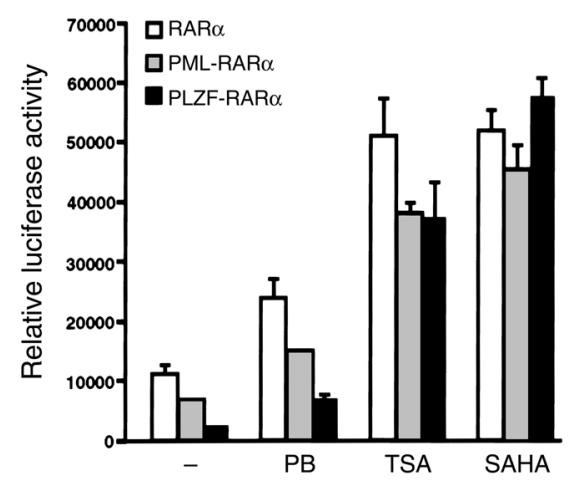

Figure 3

HDACls reverse the transcriptional repression caused by PML-RAR $\alpha$ and PLZF-RAR $\alpha$. Cos- 1 cells were transfected with an $0.8 \mu \mathrm{g}$ DR5-Luc reporter; $0.3 \mu \mathrm{g}$ of Tk- $\beta$-galactosidase internal standard; and $0.4 \mu \mathrm{g}$ of pSG5, pSG5-RAR $\alpha$, pSG5-PML-RAR $\alpha$, or pSG5-PLZF-RAR $\alpha$ expression vectors and then incubated with various HDACls in combination with $10^{-7} \mathrm{M}$ RA. (-), $10^{-7} \mathrm{M}$ RA alone. Luciferase activity was measured in a Luminometer (Turner Designs, Sunnyvale, California, USA) and normalized with the internal $\beta$-galactosidase standard. Values represent the mean \pm SD from one of three independent transfections performed in triplicate.

nation of RA and SAHA, but not induced by either agent alone (Table 2). Representatives of the different classes of induced genes are shown. The classes include (a) genes not induced by RA, but induced by SAHA alone and RA+SAHA, such as p21 WAF1 (Figure 2); (b) genes not induced by SAHA alone, but induced by RA alone and RA+SAHA (TG, integrin $\beta 7$ [Int $\beta 7$ ], and G0S2; ref. 41]) (Figure 2); and (c) genes induced by RA alone, SAHA alone, and the combination (i.e., TK1) (Figure 2). The expression of a subset of the genes induced by either RA or SAHA alone at 6 hours appears to be potentiated at 15 hours with the combination of RA and SAHA (i.e., p21 ${ }^{\text {WAF1 }}$, G0S2). In summary, we have identified a subset of genes whose expression is induced only by the combination of RA and SAHA in NB4 cells. Furthermore, the expression of bona fide RAR $\alpha$-target genes is potentiated by the RA and SAHA combination.

$H D A C I$ reverse the transcriptional repression by $P M L$ $R A R \alpha$ and PLZF-RAR $\alpha$. We and others have demonstrated that both PML-RAR $\alpha$ and PLZF-RAR $\alpha$ $(\mathrm{X}-\mathrm{RAR} \alpha)$ proteins act as potent transcriptional repressors through recruitment of HDACs (22-24). We compared effects of PB, SAHA, and TSA on the transcriptional activity of X-RAR $\alpha$ and RAR $\alpha$ proteins. The three HDACIs had negligible effects, when compared with RA, on the transactivation of a DR5Luciferase reporter gene, which was repressed by the $\mathrm{X}$-RAR $\alpha$ and RAR $\alpha$ proteins (data not shown). However, in combination with RA, the three compounds stimulated from two- to 23-fold RA-dependent transactivation by X-RAR $\alpha$ and RAR $\alpha$ (Figure 3 ). In the presence of TSA or SAHA in combination with RA, PLZF-RAR $\alpha$ proteins displayed transactivation activity similar to that of RAR $\alpha$ and PML-RAR $\alpha$. SAHA-
RA combination was the most effective in overcoming the transcriptional repressive activity of X-RAR $\alpha$, and PB-RA was least effective.

That RA-refractory and RA-sensitive fusion proteins respond equally well to RA + HDACIs may also suggest that non-HDAC-based components of transcriptional repression by unliganded nuclear receptors $(42,43)$ play a minimal role in this specific cell context and assay (transient transfection of plasmid DNA in the presence of pharmacological doses of RA).

It has been reported that RA and arsenic trioxide $\left(\mathrm{As}_{2} \mathrm{O}_{3}\right)$, can induce the degradation of the PML-RAR $\alpha$ fusion protein $(44,45)$. The degradation of PML-RAR $\alpha$ by RA or $\mathrm{As}_{2} \mathrm{O}_{3}$ is accompanied by the relocalization of PML to its normal nuclear localization sites: the nuclear bodies $(44,45)$. To investigate whether HDACIs also induced changes of PML and/or PML-RAR $\alpha$ localization in NB4 cells, we performed immunofluorescence staining of NB4 cells with an anti-PML antibody, which recognizes both PML-RAR $\alpha$ and PML proteins. No effect of HDACIs alone on the localization of PMLRAR $\alpha$ or PML was observed. In addition, HDACIs in combination with RA did not alter the RA-induced restoration of PML nuclear body (data not shown). a

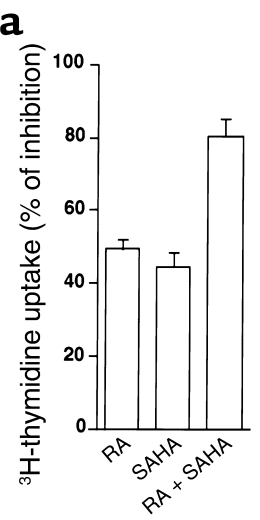

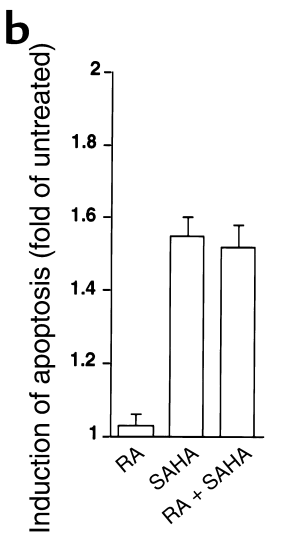

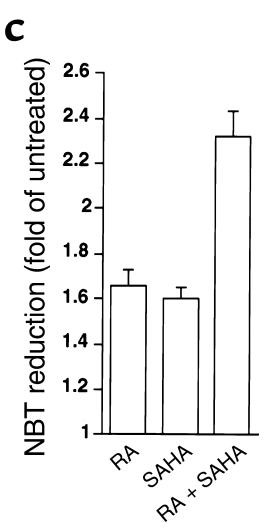

\section{Figure 4}

Effects of SAHA alone or in combination with RA on leukemic cells from $P L Z F-R A R \alpha / R A R \alpha-P L Z F$ double TM. BM cells collected from leukemic double TM were cultured at an initial cell density of $2 \times 10^{6} / \mathrm{ml}$. Each sample from the leukemic transgenic mice was divided equally and incubated in parallel with or without SAHA $(0.6 \mu \mathrm{M})$, $\mathrm{RA}\left(10^{-6} \mathrm{M}\right)$, or the combination, as indicated. Results shown here are from one of three independent experiments performed in triplicate (mean \pm SD). (a) SAHA alone causes growth inhibition and promotes $\mathrm{RA}$-induced growth inhibition. ${ }^{3} \mathrm{H}$-Thymidine incorporation rate was measured after 44 hours' culture as indicated above, with $\left[{ }^{3} \mathrm{H}\right]$ thymidine added for the final 24 hours. These cultures were maintained in the presence of $2 \%$ pokeweed mitogen-stimulated SCCM containing IL3 and GM-CSF). Results are expressed as percentage of inhibition upon treatment compared with untreated control. (b) SAHA alone causes apoptosis. TUNEL-positive cells were stained and scored after 48 hours of culture in the presence of SCCM and expressed as the induction of apoptosis upon treatment (untreated =1). (c) SAHA in combination with RA displays an additive effect on differentiation induction. NBT reduction assay was performed after 4 days of incubation as indicated and in the absence of SCCM. Results are expressed as the fold increase in $\mathrm{OD}_{540} / 10^{6}$ cells (untreated $=1$ ). 
SAHA in combination with RA induces differentiation in $B M$ cells from PLZF-RAR $\alpha / R A R \alpha-P L Z F$ leukemic mice. Given that SAHA in combination with RA converted the transcriptional repression exerted by PML-RAR $\alpha$ or PLZF-RAR $\alpha$ to transcriptional activation to a similar extent, we studied the ex vivo biologic activities of SAHA alone and in combination with RA on BM cells collected from our leukemic PLZF-RAR $\alpha / R A R \alpha-P L Z F$ double TM. In agreement with the results we obtained from NB4 cells, SAHA at a concentration of $0.6 \mu \mathrm{M}$ was able to inhibit proliferation, induce apoptosis, and increase RA-induced growth inhibition in these leukemic cells harboring the PLZF-RAR $\alpha / R A R \alpha-P L Z F$ transgenes, as evaluated by $\left[{ }^{3} \mathrm{H}\right]$ thymidine incorporation (Figure 4a), TUNEL staining (Figure 4b), and Annexin V staining (data not shown). SAHA in combination with RA significantly increased differentiation compared with either drug alone as evaluated by NBT reduction assay (Figure 4c) and morphological examination (data not shown).

SAHA induces in vivo accumulation of acetylated histones at a nontoxic dose. We administered doses of SAHA to wildtype mice and monitored the peripheral blood weekly. No toxicity was observed as evaluated by weight gain at doses of $20-100 \mu \mathrm{g} / \mathrm{gbw}$ alone or in combination with RA daily. Myelosuppression was not observed after a 28-day treatment at these doses in a 6-month follow up. We next tested whether SAHA at these doses could induce accumulation of acetylated histones in vivo. Cells were collected from leukemic TM and wild-type

\section{Figure 5}

In vivo effects of SAHA, RA, and the combination of both on PLZF$R A R \alpha / R A R \alpha-P L Z F$ double leukemic TM. (a) Western blot analysis of acetylated histone $\mathrm{H} 4$ in murine cells upon in vivo administration of SAHA. Wild-type and leukemic TM were given SAHA $(20 \mu \mathrm{g} / \mathrm{gbw})$ by intraperitoneal injection. Histones were acid extracted from murine peripheral blood, BM, and spleen cells in untreated mice and 2 hours after SAHA administration. (b) SAHA in combination with RA treatment prolongs survival in PLZF-RAR $\alpha / R A R \alpha-P L Z F$ double TM with leukemia. Upon presentation of leukemia as monitored by automatic and differentiated counts on peripheral blood samples from PLZF$R A R \alpha / R A R \alpha-P L Z F$ double TM, RA was administered daily at a dose of $1.5 \mu \mathrm{g} / \mathrm{gbw}$ for 2 weeks. The RA-treated mice were then randomly assigned into three groups: RA alone ( $1.5 \mu \mathrm{g} / \mathrm{gbw})$, SAHA (50 $\mu \mathrm{g} / \mathrm{gbw})$ alone, and SAHA ( $50 \mu \mathrm{g} / \mathrm{gbw})$ in combination with RA (1.5 $\mu \mathrm{g} / \mathrm{gbw})$. The treatment was continued for 4 weeks. During and after the treatment, the mice were bled weekly, and automatic and morphological differential counts were performed on each sample to evaluate response to treatment until each animal died. Kaplan Meier analysis was used to compare the cumulative survival period between SAHA in combination with RA $(n=11)$ and RA $(n=9)$ or SAHA $(n=6)$ alone. The black bar on the abscissa represents the 28-day period of treatment. Survival time reflects the days from the initiation of therapies until death for each mouse. (c) Complete remission was induced by SAHA + RA treatment in six of 11 leukemic PLZF$R A R \alpha / R A R \alpha-P L Z F$ double transgenic mice. Analyses were performed weekly as described above. SAHA + RA causes the same duration of disease-free survival (time in remission) in PLZF-RAR $\alpha / R A R \alpha-P L Z F$ leukemic mice as that achieved in $P M L-R A R \alpha$ transgenic leukemic mice treated with RA $(1.5 \mu \mathrm{g} / \mathrm{gbw})$.
}

mice 2 hours after intraperitoneal injection of SAHA, and histones were isolated for Western blot analysis from peripheral blood, BM, and spleen cells. Acetylated histones were undetectable in extracts isolated from cells of untreated mice (Figure 5a). SAHA (20 $\mu \mathrm{g} / \mathrm{gbw})$ induced accumulation of acetylated histone $\mathrm{H} 4$ in peripheral blood, BM, and spleen cells from both wildtype mice and TM (Figure 5a and data not shown).

$S A H A$ in combination with $R A$ prolongs survival and induces complete remission in PLZF-RAR $\alpha / R A R \alpha-P L Z F$ double TM. To test the efficacy of HDACIs in the treatment of $\mathrm{t}(11 ; 17)$ APL, we performed therapeutic trials on leukemic PLZF-RAR $\alpha / R A R \alpha-P L Z F$ double TM (32). As previously described, these mice displayed the classical features of $t(11 ; 17)$ APL, die within 3 weeks after leukemia presentation and are extremely resistant to treatments such as $\mathrm{RA}, \mathrm{As}_{2} \mathrm{O}_{3}$, or their combination. This preclinical trial consisted of three arms: RA, RA+SAHA, and SAHA alone. The median disease-carrying survival period was 62 days for mice treated with RA in combination with SAHA compared with 36 days for mice treated with RA alone and with 43 days for SAHA alone. Kaplan Meier analysis showed the difference of survival period upon different treatment to be statistically significant $(P<0.05)$ (Figure $5 b)$. Remission was considered to be achieved if on at least two consecutive analyses, the peripheral blood counts and hemoglobin were: $\mathrm{WBC}<25 \times 10^{3} / \mathrm{ml}$; Myeloid cells $<30 \%$; Disappearance of immature cells; Plt $>800 \times 10^{3} / \mathrm{ml}$ and $\mathrm{Hb}>10 \mathrm{~g} / \mathrm{dl}$. Six of 11 (54.6\%) of the mice treated with

$\mathbf{a}$

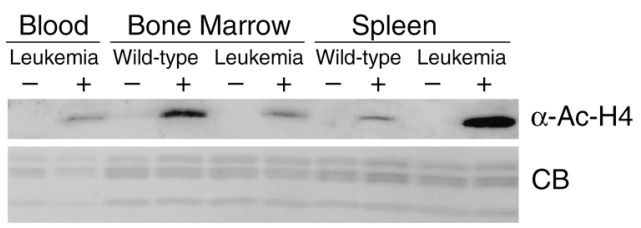

b

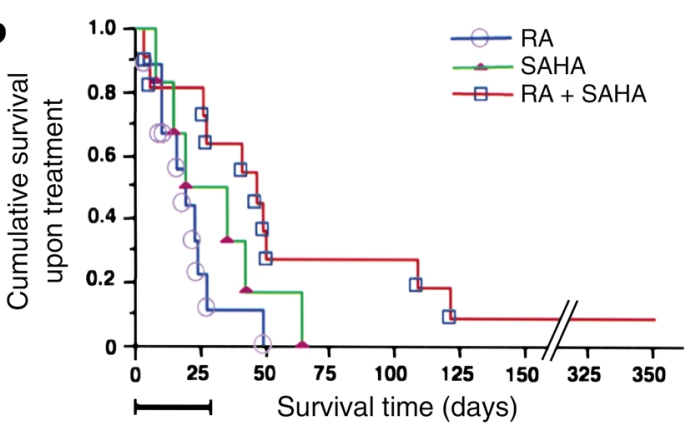

C

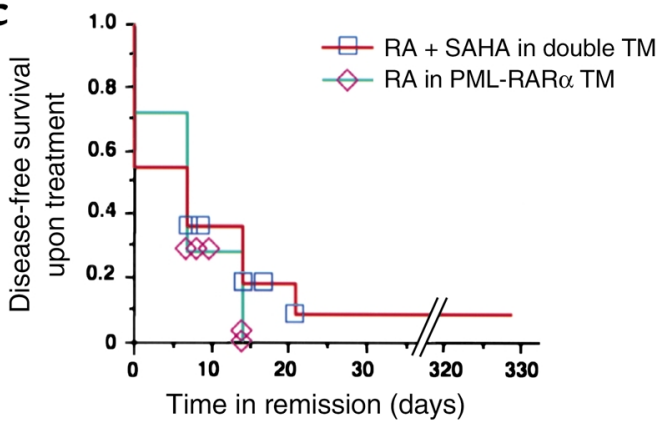


SAHA in combination with RA achieved a complete remission, which lasted 1-7 weeks (Figure 5c). No mice treated with RA alone or SAHA alone achieved remission. The duration of the remission induced by SAHA and RA in the PLZF-RAR $\alpha / R A R \alpha-P L Z F$ double TM, is the same as, if not better than, that induced by RA (at the same dose) in PML-RAR $\alpha$ TM (Figure 5d; ref. 22). Therefore, the combination of RA with SAHA not only results in longer survival but can lead to disease remission in PLZF-RAR $\alpha / R A R \alpha-P L Z F$ double TM.

\section{Discussion}

The X-RAR $\alpha$ fusion proteins of APL exert their leukemogenic role through HDAC-dependent aberrant transcriptional repression and chromatin remodeling (22-24). Thus, APL cell line and transgenic mice represent a model system to test the biologic activity of HDACIs. PLZF-RAR $\alpha / R A R \alpha-P L Z F$ double TM are particularly appropriate for the study because they represent faithful models of APL and mimic the therapeutic unresponsiveness of human $\mathrm{t}(11 ; 17)$ APL to several conventional (RA and chemotherapy) (17) and experimental $\left(\mathrm{As}_{2} \mathrm{O}_{3}\right)$ therapeutic approaches (35). This study examined the effects of three HDACIs: TSA, SAHA, and $\mathrm{PB}$ in the cells harboring $\mathrm{t}(15 ; 17)$ and primary $\mathrm{BM}$ cells from PLZF-RAR $\alpha / R A R \alpha-P L Z F$ double TM. We show that each can inhibit growth, induce apoptosis, and potentiate the differentiating and growth inhibitory activity of RA. Further, we demonstrate for the first time in vivo that the combination of RA and SAHA can increase survival and induce remission in APL transgenic mice refractory to RA treatment. We term this therapeutic approach "transcription therapy," by which specific targeting of the aberrant transcription complex renders it possible to antagonize the activity of oncogenic factors (46). Altogether, our findings strongly suggest that RA and SAHA in combination could be effectively utilized for the treatment of APL at presentation, both in $\mathrm{t}(15 ; 17) \mathrm{RA}$-sensitive as well as in $\mathrm{t}(11 ; 17) \mathrm{RA}-$ resistant APL. We have previously reported that RA plus $\mathrm{PB}$ could induce a complete remission in a 13 -year-old child with $\mathrm{t}(15 ; 17)$ APL that had become refractory to RA. This remission was sustained with repeated courses of therapy for 8 months before the patient developed resistance to this combination therapy (ref. 31; R.P. Warrell, Jr., and P.P. Pandolfi, unpublished observation). Four other patients with $\mathrm{t}(15 ; 17) \mathrm{RA}$-refractory APL failed to respond to combination therapy (32). It is possible that RA plus SAHA may have greater potential than $\mathrm{RA}$ plus $\mathrm{PB}$, for the treatment of APL patients that develop resistance to treatment with RA alone. It must be remembered, however, that while there is a straightforward mechanistic rationale for treating APL at presentation with HDACIs plus RA, this rationale is lacking in treating acquired RA-refractory APL, in view of the fact that the mechanisms of this acquired resistance are not fully elucidated and might be heterogeneous. The only molecular findings that correlate with acquired RA resistance in
APL are mutations in the ligand binding domain of the PML-RAR $\alpha$ fusion protein. These mutations have been found only in a subset of these RA-refractory APL patients $(25 \%)$. The mechanisms by which RA resistance occurs in the remaining $75 \%$ of cases are not known. On the basis of our findings, one could speculate, for instance, that the $25 \%$ of APL that harbor the mutation in the PML-RAR $\alpha$ fusion protein may not respond to HDACIs + RA, as the RA binding is required for the recruitment of the coactivator complex.

The finding that the combination of RA and HDACIs, but neither alone, is effective in treating leukemia in the TM, is consistent with a model in which PLZF-RAR $\alpha$, in the presence of pharmacological doses of RA, can recruit histone acetyltransferases through the RAR $\alpha$ moiety and, at the same time, HDAC through the PLZF NH${ }_{2}$-moiety (22-24).

HDAC activity is required at least in part for transcriptional repression by other transcription factors potentially relevant for tumor suppression and cellular differentiation such as $\mathrm{Rb}$ and the Mad/Max complex (47-51). Thus, in principle, HDACIs may affect these pathways as well. However, X-RAR $\alpha$ proteins may contribute to leukemogenesis also by sequestration of components of the corepressor/HDAC complex, in turn impairing $\mathrm{Rb}$ and $\mathrm{Mad} / \mathrm{Max}$ repressive activity. In this scenario, HDACIs would primarily antagonize the repression by the fusion oncoproteins. Although the precise mechanisms by which HDACIs in combination with RA antagonize the aberrant function of the X-RAR $\alpha$ oncoproteins remain to be elucidated, we have shown in this report that therapy with RA+HDACIs is effective.

It is conceivable that treatment with HDACIs can be applied to other forms of cancers in which HDACdependent aberrant transcriptional repression is implicated as a pathogenic mechanism. Examples are nonHodgkin's lymphomas, which are associated with structural alterations disrupting the BCL6 proto-oncogene (52-55), and M2 subtype of myeloid leukemia associated with chromosomal translocation involving the AML1 and ETO genes $(56,57)$. Both BCL6 and the AML1-ETO fusion protein are transcriptional repressors, which can recruit nuclear receptor corepressors and histone deacetylases $(54,56,57)$. In vitro histone deacetylases inhibitors have recently been shown to restore RA transcriptional activation and induce the differentiation of primary blasts from AML patients (58). In addition, HDACIs exert a dramatic growth inhibitory and proapoptotic activity in leukemic cell lines lacking the X-RAR $\alpha$ fusion proteins such as the myeloid leukemia cell lines U937 (59) and HL60 (data not shown). Inhibition of proliferation and induction of apoptosis by HDACIs are also potentiated by RA. As in APL cells, HDACIs increase RA-induced differentiation (data not shown). Furthermore, differentiating or growth inhibitory and/or proapoptotic activity by HDACIs has been recently reported in several tumor cell lines $(28,60-65)$ as well as in several tumor-bearing animal models including prostate, breast, and lung 
cancers $(32,61,65,66)$. Preliminary analysis of the transcriptional consequences of exposure to HDACIs revealed that only a finite subset of genes is modulated upon treatment (64). Here, we demonstrate that indeed SAHA potentiates the expression of bona fide RAR $\alpha$ target genes such as $\mathrm{p} 21^{\mathrm{WAF}}$ and transglutaminase 2 . Additionally, a small subset of genes $(0.05 \%)$ is induced only by the combination of RA and SAHA. It remains to be seen whether these are also direct bone fide RAR $\alpha$ target genes. However, RA and SAHA also induce distinct subsets of genes in NB4 cells. In fact, although 3\% of the induced genes are induced by both RA alone and SAHA alone, the majority of genes induced by coculture with RA and SAHA are the result of the expression of both subsets of genes. These results indicate that primary action of SAHA may be independent of X-RAR $\alpha$ in APL. Taken together, the present data are consistent with a model by which HDACIs specifically derepress a preprogrammed set of genes whose transcriptional activation induces cell-cycle arrest, apoptosis, and/or cell differentiation and can enhance the expression of RA target genes. These findings support the proposal that HDACIs alone or in combination with RA might have utility as anticancer agents.

\section{Acknowledgments}

This work was supported in part by NCI grant CA0974823, NIH grant CA-71692 (to P.P. Pandolfi), grant UO1 CA-84292 (awarded to P.P. Pandolfi and V.M. Richon), and the De Witt Wallace Fund for Memorial Sloan-Kettering Cancer Center. L.-Z. He was partially supported by the Charles H. Revson Foundation. P.P. Pandolfi is a Scholar of the Leukemia-Lymphoma Society of America (formerly known as the Leukemia Society of America). Memorial Sloan-Kettering Cancer Center and Columbia University jointly hold the patents on the hydroxamic acid hybrid polar compounds, including SAHA, which are exclusively licensed to Aton Pharma, Inc. of which Richard A. Rifkind, Paul A. Marks and Victoria M. Richon are founders. Both institutions and the founders have an equity position in Aton Pharma, Inc.

1. Warrell, R.P., Jr., de Thé, H., Wang, Z.Y., and Degos L. 1993. Acute promyelocytic leukemia. N. Engl. J. Med. 329:177-189.

2. Melnick, A., and Licht, J.D. 1999. Deconstructing a disease: RARalpha, its fusion partners, and their roles in the pathogenesis of acute promyelocytic leukemia. Blood. 93:3167-3215.

3. He, L.-Z., Merghoub, T., and Pandolfi, P.P. 1999. In vivo analysis of the molecular pathogenesis of acute promyelocytic leukemia in the mouse and its therapeutic implications. Oncogene. 18:5278-5292.

4. Pandolfi, P.P., et al. 1991. Structure and origin of the acute promyelocytic leukaemia myl/RAR $\alpha$ cDNA and characterization of its retinoid-binding and transactivation properties. Oncogene. 6:1285-1292.

5. de Thé, H., et al. 1991. The PML/RAR $\alpha$ fusion mRNA generated by the $\mathrm{t}(15 ; 17)$ translocation in acute promyelocytic leukemia encodes a functionally altered RAR. Cell. 66:675-684.

6. Kakizuka, A., et al. 1991. Chromosomal translocation $\mathrm{t}(15 ; 17)$ in human acute promyelocytic leukemia fuses RAR $\alpha$ with a novel putative transcription factor, PML. Cell. 66:663-674.

7. Goddard, A.D., Borrow, J., Freemont, I., and Solomon, E. 1991. Characterization of a zinc finger gene disrupted by the $\mathrm{t}(15 ; 17)$ in acute promyelocytic leukemia. Science. 254:1371-1374.

8. Chen, Z., et al. 1993. Fusion between a novel Kruppel-like zinc finger gene and the retinoic acid receptor- $\alpha$ locus due to a variant $t(11 ; 17)$ transloca- tion associated with acute promyelocytic leukaemia. EMBO J. 12:1161-1167.

9. Chen, S.-J., et al. 1993. Rearrangements of the retinoic acid receptor alpha and promyelocytic leukemia zinc finger genes resulting from $\mathrm{t}(11 ; 17)(\mathrm{q} 23 ; 21)$ in a patient with acute promyelocytic leukemia. J. Clin. Invest. 91:2260-2267.

10. Redner, R.L., Rush, E.A., Faas, S., Rudert, W.A., and Corey, S.J. 1996. The $t(15 ; 17)$ variant of acute promyelocytic leukemia expresses a nucleophosmin-retinoic acid receptor fusion. Blood. 87:882-886.

11. Wells, R.A., Catzavelos, C., and Kamel-Reid, S. 1997. Fusion of retinoic acid receptor alpha to NuMA, the nuclear mitotic apparatus protein, by a variant translocation in acute promyelocytic leukaemia. Nat. Genet. 17:109-113.

12. Arnould, C., et al. 1999. The signal transducer and activator of transcription STAT5b gene is a new partner of retinoic acid receptor alpha in acute promyelocytic-like leukemia. Hum. Mol. Genet. 8:1741-1749.

13. Warrell, R.P., Jr., et al. 1991. Differentiation therapy of acute promyelocytic leukemia with tretinoin (all-trans-retinoic acid). N. Engl. J. Med. 324:1385-1393.

14. Soignet, S.L., et al. 1998. Complete remission after treatment of acute promyelocytic leukemia with arsenic trioxide. N. Engl. J. Med. 339:1341-1348.

15. Licht, J.D., et al. 1995. Clinical and molecular characterization of a rare syndrome of acute promyelocytic leukemia associated with translocation (11;17). Blood. 85:1083-1094.

16. Chambon, P. 1996. A decade of molecular biology of retinoic acid receptors. FASEB J. 10:940-954.

17. Urnov, F.D., Wolffe, A.P., and Guschin D. 2001. Molecular mechanisms of corepressor function. Curr. Top. Microbiol. Immunol. 254:1-33.

18. Rietveld, L.E.G., Caldenhoven, E., and Stunnenberg, H.E. 2001. Avian erythroleukemia: a model for corepressor function in cancer. Oncogene. 20:3100-3109.

19. Grunstein, M. 1997. Histone acetylation in chromatin structure and transcription. Nature. 389:349-352.

20. Gudas, L.J., Sporn, M.B., and Roberts, A.B. 1994. Cellular biology and biochemistry of the retinoids. In The retinoids. M.B. Sporn, A.B. Roberts, D.S. Goodman, editors. Raven Press. New York, New York, USA. 443-520.

21. Smith, M.A., Parkinson, D.R., Cheson, B.D., and Friedman, M.A. 1992. Retinoids in cancer therapy. J. Clin. Oncol. 10:839-864.

22. He, L.-Z., et al. 1998. Distinct interactions of PML-RARalpha and PLZFRARalpha with co-repressors determine differential responses to RA in APL. Nat. Genet. 18:126-135.

23. Grignani, F., et al. 1998. Fusion proteins of the retinoic acid receptoralpha recruit histone deacetylase in promyelocytic leukaemia. Nature. 391:815-818.

24. Lin, R.J., et al. 1998. Role of the histone deacetylase complex in acute promyelocytic leukaemia. Nature. 391:811-814.

25. He, L.-Z., et al. 1997. Acute leukemia with promyelocytic features in PML/RAR $\alpha$ transgenic mice. Proc. Natl. Acad. Sci. USA. 94:5302-5307.

26. Kruh, J. 1982. Effects of sodium butyrate, a new pharmacological agent, on cells in culture. Mol. Cell. Biochem. 42:65-82.

27. Yoshida, M., Horinouchi, S., and Beppu, T. 1995. Trichostatin A and trapoxin: novel chemical probes for the role of histone acetylation in chromatin structure and function. Bioessays. 17:423-430.

28. Richon, V.M., et al. 1998. A class of hybrid polar inducers of transformed cell differentiation inhibits histone deacetylases. Proc. Natl. Acad. Sci. USA. 95:3003-3007.

29. Nakajima, H., Kim, Y.B., Terano, H., Yoshida, M., and Horinouchi, S. 1998. FR901228, a potent antitumor antibiotic, is a novel histone deacetylase inhibitor. Exp. Cell Res. 241:126-133.

30. Darkin-Rattray, S.J., et al. 1996. Apicidin: a novel antiprotozoal agent that inhibits parasite histone deacetylase. Proc. Natl. Acad. Sci. USA. 93:13143-13147.

31. Warrell, R.P., Jr., He, L.-Z., Richon, V., Calleja, E., and Pandolfi, P.P. 1998. Therapeutic targeting of transcription in acute promyelocytic leukemia by use of an inhibitor of histone deacetylase. J. Natl. Cancer Inst. 90:1621-1625.

32. Novich, S., et al. 1999. Initial clinical evaluation of transcription therapy for cancer: all-trans retinoic acid plus phenylbutyrate. Blood. 94:(Suppl. 1)61a. (Abstr.)

33. Marks, P.A., Richon, V.M., and Rifkind, R.A. 2000. Histone deacetylase inhibitors J. Natl. Cancer Inst. 92:1210-1216.

34. He, L.-Z., et al. 2000. Two hits are critical for promyelocytic leukemia. Mol. Cell. 6:1131-1141.

35. Rego, E.M., He, L.-Z., Warrell, R.P., Jr., Wang, Z.-G., and Pandolfi, P.P. 2000. RA and $\mathrm{As}_{2} \mathrm{O}_{3}$ treatment in transgenic models of APL unravel the distinct nature of the leukemogenic process induced by the PML-RAR $\alpha$ and PLZF-RAR $\alpha$ oncoproteins. Proc. Natl. Acad. Sci. USA. 97:10173-10178.

36. Vermes, I., Haanen, C., Steffens-Nakken, H., and Reutelingsperger, C. 1995. A novel assay for apoptosis. FlVow cytometric detection of phosphatidylserine expression on early apoptotic cells using fluorescein labeled annexin V. J. Immunol. Methods. 184:39-51. 
37. Chomczynski, P., and Sacchi, N. 1987. Single-step method of RNA isolation by acid guanidinium thiocyanate-phenol-chloroform extraction. Anal. Biochem. 162:156-159.

38. el-Diery, W.S., et al. 1993. WAF1, a potential mediator of p53 tumor suppression. Cell. 75:817-825.

39. Zhou, X., Richon, V.M., Ngo, L., Rifkind, R.A., and Marks, P.A. 1999. Cloning of the cDNA encoding phenylalanyl tRNA synthetase regulatory alpha-subunit-like protein whose expression is down-regulated during differentiation. Gene. 233:13-19.

40. Benedetti, L., et al. 1996. Retinoid-induced differentiation of acute promyelocytic leukemia involves PML-RARalpha-mediated increase of type II transglutaminase. Blood. 87:1939-1950.

41. Tamayo, P., et al. 1999. Interpreting patterns of gene expression with selforganizing maps: methods and application to hematopoietic differentiation. Proc. Natl. Acad. Sci. USA. 96:2907-2912.

42. Fondell, J.D., Brunel, F., Hisatake, K., and Roeder, R.G. 1996. Unliganded thyroid hormone receptor alpha can target TATA-binding protein for transcriptional repression. Mol. Cell. Biol. 16:281-287.

43. Urnov, F.D., et al. 2000. Targeting of N-CoR and histone deacetylase 3 by the oncoprotein v-erbA yields a chromatin infrastructure-dependent transcriptional repression pathway. EMBOJ. 19:4074-4090.

44. Look, A.T. 1998. Arsenic and apoptosis in the treatment of acute promyelocytic leukemia. J. Natl. Cancer Inst. 90:86-88.

45. Wang, Z.G., et al. 1998. Arsenic trioxide and melarsoprol induce programmed cell death in myeloid leukemia cell lines and function in a PML and PML-RARalpha independent manner. Blood. 92:1497-1504.

46. Pandolfi, P.P. 2001. Transcription therapy for cancer. Oncogene. 20:3116-3127.

47. Heinzel, T., et al. 1997. A complex containing $\mathrm{N}-\mathrm{CoR}, \mathrm{mSin} 3$ and histone deacetylase mediates transcriptional repression. Nature. 387:43-48.

48. Alland, L., et al. 1997. Role for N-CoR and histone deacetylase in Sin3mediated transcriptional repression. Nature. 387:49-55.

49. Brehm, A., et al. 1998. Retinoblastoma protein recruits histone deacetylase to repress transcription. Nature. 391:597-601.

50. Magnaghi-Jaulin, L., et al. 1998. Retinoblastoma protein represses transcription by recruiting a histone deacetylase. Nature. 391:601-605.

51. Luo, R.X., Postigo, A.A., and Dean, D.C. 1998. Rb interacts with histone deacetylase to repress transcription. Cell. 92:463-473.

52. Lo Coco, F., et al. 1994. Rearrangements of the BCL-6 gene in diffuse large-cell non-Hodgkins lymphoma. Blood. 83:1757-1759.

53. Magrath, I. 1990. Lymphocyte ontogeny: a conceptual basis for understanding neoplasia of the immune system. In The non-Hodgkin's lymphoma. I. Magrath, editor. Williams and Wilkins. Baltimore, Maryland, USA. $29-48$
54. Dhordain, P., et al. 1997. Corepressor SMRT binds the BTB/POZ repressing domain of the LAZ3/BCL6 oncoprotein. Proc. Natl. Acad. Sci. USA. 94:10762-10767.

55. Ye, B.H., et al. 1995. Chromosomal translocations cause deregulated BCL6 expression by promoter substitution in B-cell lymphoma. EMBO J. 14:6209-6217.

56. Wang, J., Hoshino, T., Redner, R.L., Kajigaya, S., and Liu, J.M. 1998. ETO, fusion partner in $\mathrm{t}(8 ; 21)$ acute myeloid leukemia, represses transcription by interaction with the human $\mathrm{N}-\mathrm{CoR} / \mathrm{mSin} 3 / \mathrm{HDAC} 1$ complex. Proc. Natl. Acad. Sci. USA. 95:10860-10865.

57. Gelmetti, V., et al. 1998. Aberrant recruitment of the nuclear receptor corepressor-histone deacetylase complex by the acute myeloid leukemia fusion partner ETO. Mol. Cell. Biol. 18:7185-7191.

58. Ferrara, F.F., et al. 2001. Histone deacetylase-targeted treatment restores retinoic acid signaling and differentiation in acute myeloid leukemia. Cancer Res. 61:2-7.

59. Vrana, J.A., et al. 1999. Induction of apoptosis in U937 human leukemia cells by suberoylanilide hydroxamic acid (SAHA) proceeds through pathways that are regulated by $\mathrm{Bcl}-2 / \mathrm{Bcl}-\mathrm{XL}$, c-Jun, and $\mathrm{p} 21 \mathrm{CIP} 1$, but independent of p53. Oncogene. 18:7016-7025

60. Kim, Y.B., Lee, K.H., Sugita, K., Yoshida, M., and Horinouchi, S. 1999. Oxamflatin is a novel antitumor compound that inhibits mammalian histone deacetylase. Oncogene. 18:2461-2470.

61. Cohen, L.A., et al. 1999. Chemoprevention of carcinogen-induced mammary tumorigenesis by the hybrid polar cytodifferentiation agent, suberanilohydroxamic acid (SAHA). Anticancer Res. 19:4999-5006.

62. Sambucetti, L.C., et al. 1999. Histone deacetylase inhibition selectively alters the activity and expression of cell cycle proteins leading to specific chromatin acetylation and antiproliferative effects J. Biol. Chem. 274:34940-34947.

63. Saito, A., et al. 1999. A synthetic inhibitor of histone deacetylase, MS-27275 , with marked in vivo antitumor activity against human tumors. Proc. Natl. Acad. Sci. USA. 96:4592-4597.

64. Van Lint, C., Emiliani, S., and Verdin, E. 1996. The expression of a small fraction of cellular genes is changed in response to histone hyperacetylation. Gene Expr. 5:245-253.

65. Butler, L.M., et al. 2000. Suberoylanilide hydroxamic acid (SAHA), an inhibitor of histone deacetylase, suppresses the growth of prostate cancer cells in vitro and in vivo. Cancer Res. 60:5165-5170.

66. Desai, D., El-Baoumy, K., and Amin, S. 1999. Chemopreventive efficacy of suberanilohydroxamic acid (SAHA), a cytodifferentiating agent, against tobacco-specific nitrosamine 4-(methylnitro-amino)-1-(3pyridyl)-1butanone (NNK)-induced lung tumorigenesis. Proc. Annu. Meet. Am. Assoc. Cancer Res. 40:28. (Abstr.) 